\title{
Down-regulation of Notch-1 expression decreases PU.1-mediated myeloid differentiation signaling in acute myeloid leukemia
}

\author{
PO-MIN CHEN ${ }^{1,2}$, CHUEH-CHUAN YEN ${ }^{1,2}$, WEI-SHU WANG ${ }^{1,2}$, YU-JEN LIN ${ }^{1}$, CHIAU-JUN CHU ${ }^{1,2}$ \\ TZEON-JYE CHIOU ${ }^{1,2}$, JIN-HWANG LIU ${ }^{1,2}$ and MUH-HWA YANG ${ }^{1,3}$ \\ ${ }^{1}$ Division of Hematology-Oncology, Department of Medicine, Taipei Veterans General Hospital, Taipei; \\ ${ }^{2}$ Faculty of Medicine, ${ }^{3}$ Institute of Clinical Medicine, School of Medicine, \\ National Yang-Ming University, Taipei, Taiwan R.O.C.
}

Received February 7, 2008; Accepted March 18, 2008

\begin{abstract}
The Notch receptor-mediated signaling pathways control cell fate in many types of organisms including neurogenesis, myogenesis and hematopoiesis in mammalian species. During normal hematopoiesis, Notch-1 promotes myeloid differentiation through up-regulation of the transcriptional factor PU.1. We therefore speculated that down-regulation of Notch-1 expression might be involved in the leukemogenesis of acute myeloid leukemia (AML). Here we investigated Notch-1 expression and its association with PU.1-mediated differentiation signaling in AML. The transcriptional level of Notch-1 and PU.1 was evaluated in 6 AML cell lines and 54 AML patient samples using real-time PCR analysis, and Western blot analysis of Notch-1, PU.1 and one of its downstream targets, the M-CSF receptor (MCSFR), was performed to test for confirmation. A significant decrease in the transcription levels of Notch-1 was noted in AML cell lines and patient samples, and decreased Notch-1 protein expression in AML was confirmed by Western blotting. Down-regulation of Notch-1 expression was associated with a decrease in PU.1/MCSFR expression in AML. Co-immunoprecipitation experiments showed that partial disruption of the Notch-1/ PU.1 complex was noted in AML cells. No detectable mutation of Notch-1 (ANK, PEST) and PU.1 (PEST, DBD) was noted by PCR-single-strand conformation polymorphism (SSCP) assay. These results suggest that down-regulation of Notch-1 expression decreases PU.1/MCSFR expression and disrupts the Notch-1/PU.1 complex, which may impede the PU.1-mediated myeloid signaling and contribute to the leukemogenesis of AML.
\end{abstract}

Correspondence to: Dr Muh-Hwa Yang, Division of HematologyOncology, Department of Medicine, Taipei Veterans General Hospital, No. 201, Section 2, Shih-Pai Road, Taipei, Taiwan R.O.C. E-mail: mhyang2@vghtpe.gov.tw

Key words: acute myeloid leukemia, differentiation, Notch-1, PU.1

\section{Introduction}

The Notch genes encode a group of highly preserved transmembrane receptors that transmit signals affecting the development of organisms (1-8). The Notch receptor is composed of an extracellular ligand-binding domain (ECD) and an intracellular domain (ICD) for signal transduction. Notch signaling occurs as a result of cell-cell interaction between the Notch receptor on the target cell and the ligand on the adjacent cell, and involves the proteolytic cleavage and translocation of the ICD into the nucleus (9). In humans, the Notch signaling pathway is involved in the development of the neural, muscular and lymphoid system $(8,10-13)$. Dysregulation of Notch expression has been found to contribute to several human diseases. The most striking example is T-cell lymphoid malignancy (14-16).

Hematopoiesis involves a tightly regulated series of molecular events, whereupon lineage commitment or selfrenewal of stem cells may result from intrinsic and extrinsic stochastic processes. Studies have explored the potential role of the Notch signaling pathway in hematopoiesis; both Notch-1 and -2 were detected in murine marrow precursors (Lin-Sca- ${ }^{+} \mathrm{c}-\mathrm{kit}^{+}$), and the Jagged-1 ligand was observed in murine bone marrow stroma and stromal cell lines (17). Cellcell interaction between hematopoietic and stromal cells via Notch receptor-ligand conjugation may influence cell fate and the self-renewing process of the hematopoietic system (18). The classic view holds that Notch signaling keeps cells in an undifferentiated state. It is believed that Notch activity promotes hematopoietic progenitor self-renewal, and is involved in the control of cell fate, differentiation, proliferation and apoptosis (19). However, this viewpoint changed after it was observed that Notch-1 signaling induced multilineage myeloid differentiation through direct increase of the mRNA level of the transcriptional factor PU.1 which mediates myeloid differentiation (20). We therefore speculated that decreased Notch-1 expression may participate in the leukemogenesis of human myeloid malignancies.

Acute myeloid leukemia (AML) is a disease that originates from the dysregulation of myelopoiesis, and the role of the Notch signaling system in the leukemogenesis of AML is of great interest. At present, there are only limited studies 
regarding abnormal Notch expression in AML. Notch-1 and Jagged-1 protein have been reported to be expressed in AML samples using immunoblotting (21), and Notch-1/-2 mRNA expression was detected in an AML cell line TMD7 (22). However, the role of Notch-mediated myeloid differentiation in AML has never been explored. We therefore aimed to delineate the role of Notch-mediated differentiation signaling in AML. In this study, we demonstrated a significant decrease of Notch-1/PU.1/M-CSF receptor (MCSFR) in AML cell lines and patient samples. Partial disruption of the Notch-1/PU.1 complex was shown in AML cells by co-immunoprecipitation experiments. No detectable mutation of Notch-1 (ANK, PEST) and PU.1 (PEST, DBD) was noted by PCR-single-strand conformation polymorphism (SSCP) assay. These results suggest that down-regulation of Notch-1 expression decreases the PU.1-mediated differentiation signaling and disrupts the Notch-1/PU.1 complex, which may impede the PU.1-mediated myeloid signaling and contribute to the leukemogenesis of AML.

\section{Materials and methods}

Patients and sample collection. From January 1994 to July 2001, a total of 54 patients diagnosed with AML and ten healthy donors were enrolled in this study. In accordance with the Declaration of Helsinki, informed consent was obtained in writing prior to patient and donor enrollment. All patients underwent a bone marrow examination including morphologic examination and immunohistochemical or flow cytometry study for identification of clones. Cytogenetic studies including chromosome analysis or multiplex RT-PCR (23) were performed in all patients to analyze the possible chromosomal abnormalities in AML. The heparinized bone marrow cells were harvested for further analysis. After bone marrow harvest, the mononuclear cells (MNCs) were immediately separated by sedimentation on Ficoll-Hypaque gradient. The MNCs were cryopreserved in liquid nitrogen, respectively, until use. We used the WHO classification for categorization of AML patients (24). The clinical characteristics of AML patients are listed in Table I.

Isolation of $\mathrm{CD} 4^{+}$cells as an experimental control. The CD34+ ${ }^{+}$stem cells were used as a control for Notch-1 and related signal expression in this study. Five healthy donors underwent peripheral blood stem cell collection after signing an informed consent. Stem cell purging was performed using the cell isolation device CliniMACS ${ }^{\circledR}$ on the day of stem cell harvest and for three consecutive days. During the stem cell purging procedure with CliniMACS, the cells were specifically labeled with superparamagnetic iron-dextran particles, which were covalently conjugated to the mouse anti-human CD34 antibody. After magnetic labeling, the CliniMACS system automatically passed the cells through a separation column, which was subjected to a strong permanent magnet. The magnetically labeled cells were retained in the column and separated from the unlabeled cells, which flowed through. The CliniMACS eluted the retained cells by removing the column from the magnetic field, washing the cells out, and collecting them. The CD34+ subpopulation of the purged stem cells was counted by flow cytometry.
Table I. Characteristics of the 54 AML patients.

\begin{tabular}{lc}
\hline Variables & No. $(\%)$ \\
\hline Age, years & \\
Range & $18-79$ \\
Median & 44.5 \\
Gender & \\
Male & $30(55.6)$ \\
Female & $24(44.4)$ \\
FAB classification & \\
M1 & $17(31.5)$ \\
M2 & $11(20.4)$ \\
M3 & $7(13.0)$ \\
M4 & $14(25.9)$ \\
M5 & $4(7.4)$ \\
M6 & $1 \quad(1.9)$ \\
Chromosome & \\
t(8;21) & $6(11.1)$ \\
t(15;17) & $3(5.6)$ \\
inv(16) & $3(5.6)$ \\
del(5q) & $1(1.9)$ \\
Multiple abnormalities & $5(9.3)$ \\
Normal karyotype & $36(66.7)$ \\
Response to chemotherapy & \\
Complete remission & $29(53.7)$ \\
No complete remission & $25(46.3)$ \\
\hline
\end{tabular}

AML cell lines and culture. Six AML cell lines (CTV, KG-1, RPMI-6410, MOLM13, THP1, GDM1) were cultivated under standard conditions. The cells were harvested for extraction of mRNA and protein for the subsequent analysis.

RNA extraction and real-time PCR. Total RNA was extracted from various cell samples using RNAzol B reagents (Biotech Laboratories, Houston, TX) according to the manufacturer's protocol. To eliminate potentially contaminating DNA, the RNA samples were treated with DNase (1 unit per $10 \mu \mathrm{g}$ total RNA), followed by organic solvent extraction and ethanol precipitation. Finally, the extracted RNA was dissolved in DEPC-treated sterile water, and the total RNA concentration was measured optically.

Quantitative real-time PCR for Notch-1 and PU.1 was performed in a PRISM 7700 Sequence Detection System (ABI) with the preset PCR program, and GAPDH was selected as an internal control. For Notch-1, $0.1 \mu \mathrm{g}$ of cDNA and $80 \mu \mathrm{M}$ of primers were used in the PCR reaction in 1X SYBR-Green Mixture (ABI) in a total volume of $50 \mu 1$. The target quantity was measured by identifying the threshold cycle number $\left(\mathrm{C}_{\mathrm{T}}\right)$, i.e., when the fluorescence signal crosses a preset detection threshold. GAPDH was selected as an internal control for realtime PCR experiments. Transcript levels were normalized to those of GAPDH to account for variability in the amount of cDNA in each sample, and relative expression levels were 
Table II. Nucleotide sequence of specific primers used in this study.

\begin{tabular}{cl}
\hline Study methods & \multicolumn{1}{c}{ Nucleotide sequence } \\
\hline Real-time PCR & \\
GAPDH-F & 5'-AAGGTGAAGGTCGGAGTCAACG-3' \\
GAPDH-R & 5'-CTGGAAGATGGTGATGGGATTTC-3' \\
Notch1-F & 5'-GAGCTGGACCCCATGGACGT-3' \\
Notch1-R & 5'-CTCCACGGTCTCACTCTGCA-3' \\
SSCP & \\
Notch1 ANK1-F & 5'-ACCTGCGCATGTCTGCCA-3' \\
Notch1 ANK1-R & 5'-TCGGCAGACACAGCCGCAT-3' \\
Notch1 ANK2-F & 5'-CACCCCGCTGCATGCGGC-3' \\
Notch1 ANK2-R & 5'-AGCACCTTGGCGGTCTCGT-3' \\
Notch1 ANK3-F & 5'-ACAACAGGGAGGAGACACC-3' \\
Notch1 ANK3-R & 5'-CCTTTGCTGCTGGGCTTGC-3' \\
Notch1 PEST-F & 5'-TCCCAGCCTATCATCCTTTC-3' \\
Notch1 PEST-R & 5'-TGGCTCAGACATGTGTGTC-3' \\
PU.1 PEST-F & 5'-GCTCTACCGCCACATGGAGC-3' \\
PU.1 PEST-R & 5'-CTTGCTGCCTGTCTCCCCAG-3' \\
PU.1 DBD-F & 5'-CGAGGCGGATGGCCTGGAGC-3' \\
PU.1 DBD-R & 5'-GCCGGGCGAGGGCTTAATGC-3' \\
\hline
\end{tabular}

calculated using the $2^{-\Delta \Delta \mathrm{C}_{\mathrm{T}}}$ method (25). The nucleotide sequences of primers and probes are listed in Table II.

Protein extraction, Western blotting and immunoprecipitation. A total of $50 \mu \mathrm{g}$ of cellular extracts of representative AML patient samples, CD34+ ${ }^{+}$stem cells and AML cell lines was used for Western blot analysis. After cell lysis and protein extraction, SDS-PAGE was performed, and then transferred to nitrocellulose membranes. The membranes were incubated with a polyclonal anti-Notch-1 antibody (sc-6014), anti-PU.1 antibody (sc-352), anti-MCSFR antibody (sc-692) and a polyclonal anti- $\beta$-actin antibody (sc-7210) (all from Santa Cruz Biotechnology Inc., Santa Cruz, CA) as a control for protein loading. Signals were revealed using an Amersham ECL chemiluminescence kit (Amersham Pharmacia Biotech, Piscataway, NJ).

Immunoprecipitation was performed by incubating $1000 \mu \mathrm{g}$ of whole cell extract from each AML cell line and samples with an anti-Notch-1 antibody with slow rotation for $4 \mathrm{~h}$, and the immune complexes were incubated overnight with protein A-linked agarose beads (blocked with 10\% BSA). The immunoprecipitate was washed three times with TNTG buffer $(20 \mathrm{mM}$ Tris, $\mathrm{pH} 7.5,150 \mathrm{mM} \mathrm{NaCl}, 0.1 \%$ Triton $\mathrm{X}$ $100,10 \%$ glycerol), mixed with $1 \mathrm{X}$ Laemmli dye, boiled for $10 \mathrm{~min}$ and loaded to a $12 \%$ SDS gel. After protein transfer, the filter was blocked, probed with anti-PU.1 antibody and the secondary antibody sequentially. Immunocomplexes were detected with the ECL chemiluminescence kit (Amersham Biosciences, UK). PU.1-immnoprecipitation following Western blotting was also performed to confirm the bidirectional interaction. Data are representative of two or more experiments from independent cell cultures.
PCR-SSCP analysis of Notch-1 and PU.1. To identify any mutation or deletion existing in the Notch-1 and PU.1 gene, we tested the ANK domain and the PEST region of the Notch-1 receptor at the carboxy terminus of the cytoplasmic domain, and the PEST and DNA-binding domain (DBD) of PU.1 using single-strand conformation polymorphism (SSCP) followed by direct sequence analysis. The amplified fragment was analyzed for gene mutations using the SSCP method as previously described (26). PCR product bands with a different mobility from that of the controls were excised and amplified, and the PCR products were sequenced using a dye-labeled terminator cycle sequencing kit (Perkin Elmer, Foster City, $\mathrm{CA}$ ). The nucleotide sequences of specific primers used in this study are listed in Table II.

Statistical analysis. The statistical package of the Social Sciences software (version 13.0; SPSS, Inc., Chicago, IL) was used for statistical analysis. The Pearson correlation coefficient test was used to determine the degree of correlation between Notch-1 and PU.1 mRNA expression assays, with a value $>0.6$ deemed to be a significant correlation. The independent Student's t-test was used to compare relative Notch-1 or PU.1 mRNA expression between groups. The level of statistical significance was set at 0.05 for all tests.

\section{Results}

Decreased Notch-1 mRNA and protein expression in AML cell lines and patients. To investigate the level of Notch-1 expression in AML patients and cell lines, real-time PCR of Notch-1 was performed in 6 AML cell lines and 54 AML patient samples. CD34+ hematopoietic stem cells harvested from 5 healthy donors were used as a control. A significantly decreased Notch-1 transcription level was found in all AML cell lines (mean value of $2^{-\Delta \Delta C_{T}}$ of stem cells vs. AML cell lines was $0.031 \pm 0.002$ vs. $0.018 \pm 0.014$, p $<0.001$; Fig. $1 \mathrm{~A}$ ) and patients (mean value of $2^{-\Delta \Delta \mathrm{C}_{\mathrm{T}}}$ of stem cells vs. AML samples was $0.066 \pm 0.006$ vs. $0.018 \pm 0.014$, p $<0.001$; Fig. 1B). In order to confirm the down-regulation of Notch-1 protein expression in AML, Western blot analysis was performed in six AML cell lines and representative patient samples. Decreased Notch-1 protein expression was demonstrated in AML cell lines and patient samples (Fig. 1C and D). This result indicates that down-regulation of Notch-1 mRNA and protein expression can be found in most AML cells, and may indicate that decreased Notch-1 expression may be a significant molecular event in the leukemogenesis of AML.

Down-regulation of Notch-1 expression decreased the expression of myeloid differentiation factors PU.1 and MCSFR in AML. Since Notch-1 signaling induces myeloid differentiation through up-regulation of a transcriptional factor PU.1 which regulates myeloid differentiation during normal hematopoiesis (20), we speculated that decreased Notch-1 expression in AML cells may impede the PU.1mediated myeloid differentiation pathway. In order to evaluate the association between Notch-1 expression and the PU.1-mediated myeloid differentiation pathway in AML 
A

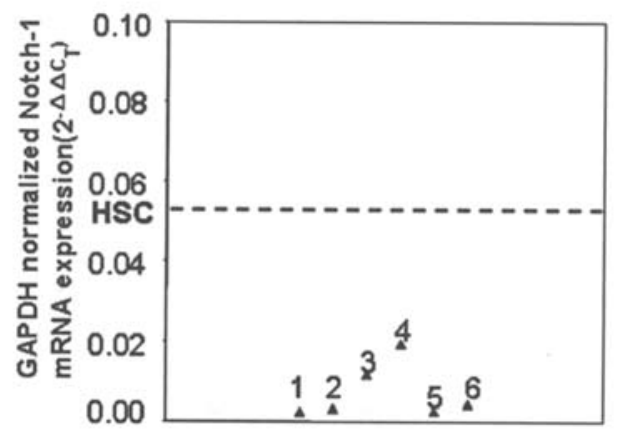

C

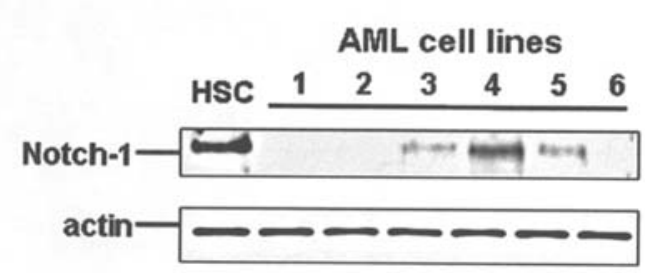

B

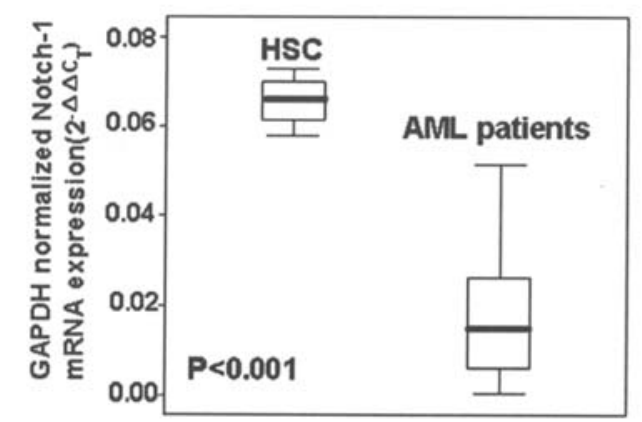

D

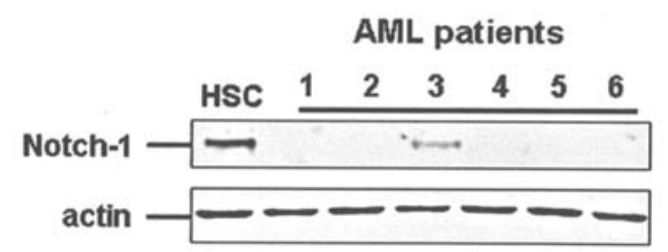

Figure 1. Down-regulation of Notch-1 expression in AML. (A) Real-time PCR results of Notch-1 expression in 54 AML patients and normal hematopoietic stem cells, and (B) real-time PCR results of Notch-1 expression in 7 AML cell lines and normal hematopoietic stem cells. (C) Western blot analysis of Notch-1 expression in AML cell lines, and (D) Western blot analysis of Notch-1 expression in the representative cases of AML.

cells, real-time RT-PCR of PU.1 was performed in AML cell lines and patient samples. Decreased PU.1 expression was found in AML cell lines (mean value of $2^{-\Delta \mathrm{C}_{\mathrm{T}}}$ of stem cells vs. AML cell lines was $0.216 \pm 0.030$ vs. $0.116 \pm 0.043, p=0.001$; Fig. 2A) and samples (mean value of $2^{-\Delta \Delta \mathrm{C}_{\mathrm{T}}}$ of stem cells vs. AML samples was $0.216 \pm 0.030$ vs. $0.141 \pm 0.074, \mathrm{p}=0.001$; Fig. 2B). A significant correlation between the transcription levels of Notch-1 and PU.1 was demonstrated (correlation coefficients of AML cell lines and samples were 0.69 and 0.66 , respectively). Western blot analysis of PU.1 and a downstream transcriptional factor of PU.1, MCSFR, was performed in the cell lines and representative patient samples to confirm the correlation between Notch-1 expression and the PU.1-mediated differentiation signaling in AML. Decreased PU.1 and MCSFR expression was demonstrated both in the AML cell lines and samples, and the expression levels of PU.1/MCSFR were correlated with Notch-1 expression (Fig. 2C and D). These results indicate that downregulation of Notch-1 expression reduces the PU.1-mediated multilineage myeloid differentiation signals in AML.

Partial disruption of the Notch-1/PU.1 complex in AML cells. We further investigated whether Notch-1 regulates myeloid differentiation not only through the transcriptional level but also by direct interaction with PU.1. To determine whether Notch-1 directly interacts with PU.1 in normal hematopoietic stem cells and whether the Notch-1/PU.1 complex is decreased in AML, immunoprecipitation of Notch-1 intracellular domain (ICD) and PU.1 was performed. As shown in Fig. 3, the direct binding of Notch-1 and PU.1 was detected in $\mathrm{CD}_{3} 4^{+}$hematopoietic stem cells. In AML cell lines and representative patient samples, decreased Notch-1/
PU.1 complex was noted. This result indicates that Notch-1 regulates myeloid differentiation not only through the transcriptional level of PU.1, but also by direct interaction with PU.1. Partial disruption of the Notch-1/PU.1 complex was found in AML cells which may reduce the PU.1-mediated myeloid differentiation that promotes leukemogenesis.

No mutation was found in the major domains of Notch-1 and PU.1 in AML. In order to test the mutational status of Notch-1 ICD and PU.1 in AML, PCR-SSCP analysis was performed in the Notch-1 intracellular ANK and PEST domain, and the PEST and DNA-binding domain (DBD) of the PU.1 of AML cell lines and representative patient samples. No notable genetic mutation was found in the Notch-1 ICD and PU.1 domains in the AML cell lines and patients (Fig. 4). This result indicates that mutation of Notch-1 and PU.1 may be a rare event and may not correlate with the leukemogenesis of human AML.

\section{Discussion}

The prevailing view of Notch signaling in myeloid hematopoiesis supposes that Notch signals function principally to inhibit the differentiation of progenitor cells and allow the expansion of the progenitor pool (28-30). However, this idea has been challenged by Schroeder et al. They reported that Notch-1 directly increases the PU.1 transcriptional level, which directs myeloid differentiation. Transiently activated Notch signaling can reduce the self-renewal of multipoint progenitors accompanied by increased and accelerated myeloid differentiation (20). The contradiction between these two hypotheses may be explained by the difference between constitutive and inducible activation of Notch; constitutive 
A

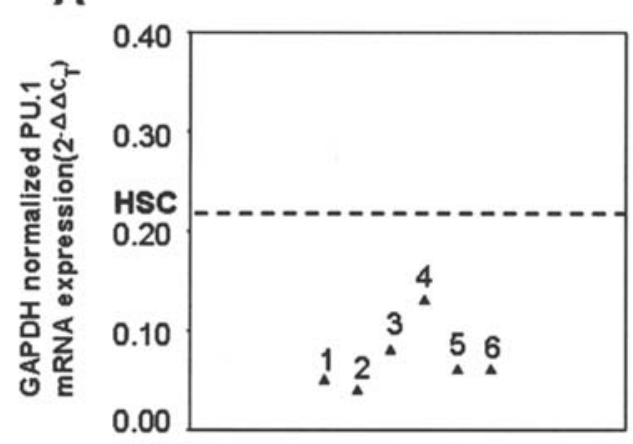

C

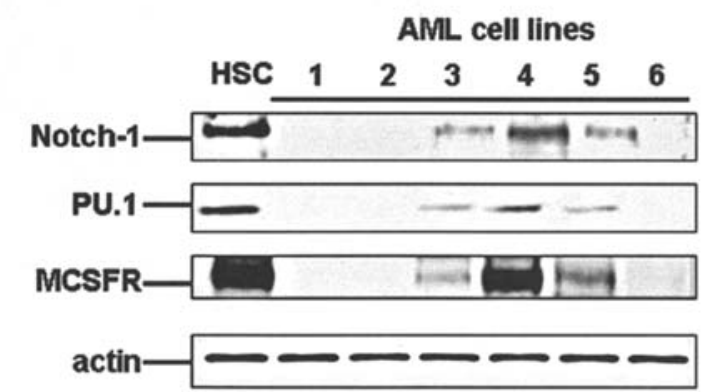

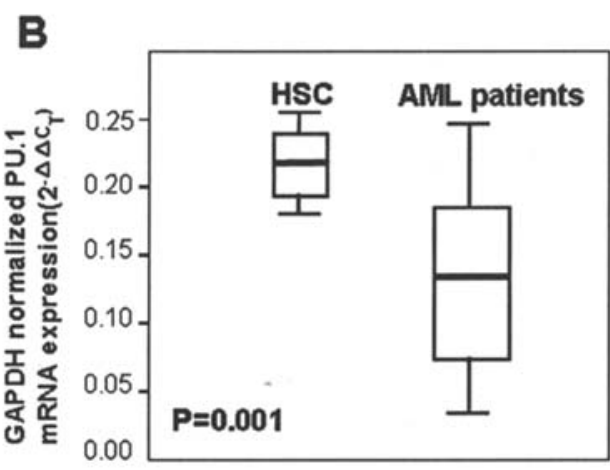

D

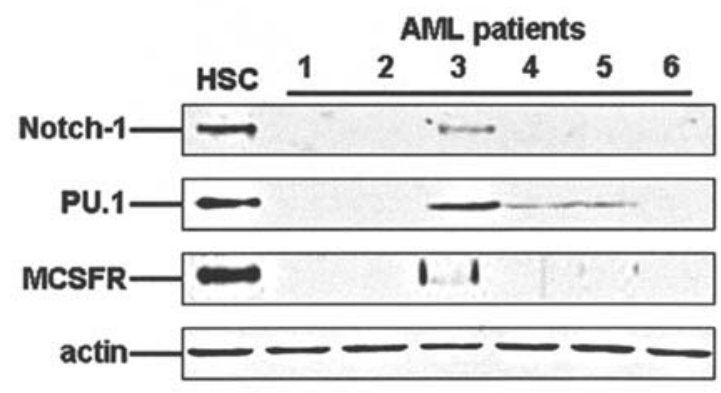

Figure 2. Down-regulation of Notch-1 expression decreased the PU.1-mediated myeloid differentiation signaling in AML. (A) Real-time PCR results of PU.1 expression in 54 AML patients and normal hematopoietic stem cells, and (B) real-time PCR results of PU.1 expression in 7 AML cell lines and normal hematopoietic stem cells. (C) Western blot analysis of Notch-1, PU.1 and MCSFR expression in AML cell lines, and (D) Western blot analysis of Notch-1, PU.1 and MCSFR expression in the representative cases of AML.

A

IP with: Notch-1

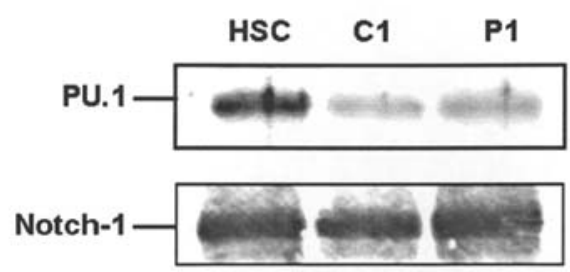

B

IP with: PU.1

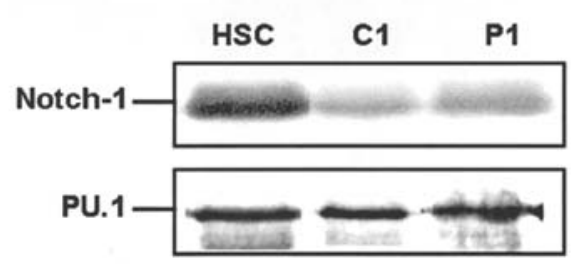

Figure 3. Interaction between Notch-1 intracellular domain and PU.1 in normal hematopoietic stem and AML cells.

activation could maintain the cells in the undifferentiated status, while inducible activation could result in myeloid differentiation. In our study, a quantitative gene assay was carried out to clarify the expression profile of the Notch signaling system in AML cell lines and primary AML cells.
A

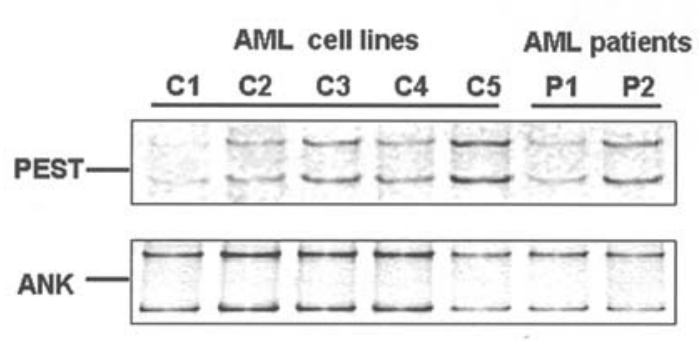

B

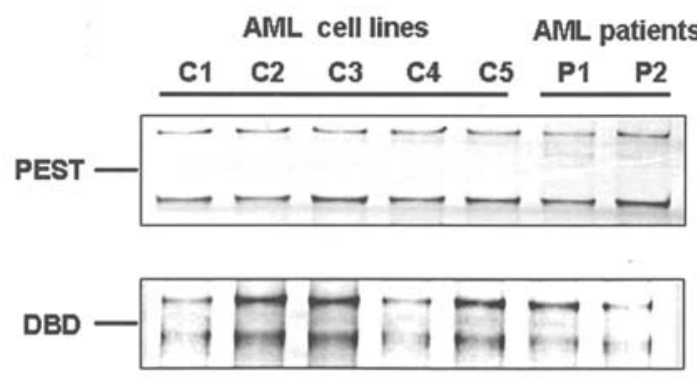

Figure 4. PCR-SSCP of domains of Notch-1 intracellular domain (ICD) and PU.1. (A) Results for the Notch-1 ICD ANK, PEST domains. (B) Results for the PU.1 PEST, DNA-binding domain (DBD).

The results revealed that Notch-1 gene expression was significantly decreased in the tested AML samples and cell lines compared with the normal hematopoietic stem cells (Fig. 1). To test whether AML cells decreased Notch-1 protein 
expression, Western blot analysis was performed and showed concordant results. According to our results, two hypotheses are addressed here to explain the role of Notch signaling in the leukemogenesis of AML. First, the inducible activation of Notch signaling mediated normal myeloid differentiation. As mentioned above, Schroeder et al reported the essential role of inducible Notch signaling in myeloid differentiation (30), and Walker et al also reported that the Notch receptors were most likely utilized by intermediate-stage granulocytes, which indicated that normal Notch expression was mandatory for the maintenance of normal granulocyte formation (31). Down-regulation of Notch expression may cause the blockage of myeloid differentiation and participation in the leukemogenesis of myeloid leukemia. Second, regarding the regulation of cell fate and programmed cell death of the Notch gene, Morimura et al reported that Notch-1 signaling could induce B-cell apoptosis (32), and Schroeder et al also showed that mNotch-1 signaling reduced the proliferation of myeloid progenitor cells by altering cell cycle kinetics (33). According to the viewpoint mentioned above, down-regulation of Notch-1 expression in myeloid cells might lead to the blockage of differentiation and may participate in the development of AML. To the best of our knowledge, our study is the first to investigate the involvement of the Notch system in a large series of AML patients.

During normal myeloid differentiation, a high level of transcriptional factor PU.1 activates promoters specific to myeloid cells, such as the M-CSF receptor (MCSFR) which controls differentiation. Since Notch-1 expression is downregulated in AML, we speculate that Notch-1-mediated myeloid differentiation signaling may be decreased in AML. In our study, significantly decreased PU.1 mRNA expression was found in AML patients (Fig. 2A) and cell lines (data not shown). Western blot analysis also revealed that the PU.1 and MCSFR protein levels were reduced in AML patients and cell lines (Fig. 2B and C). Based on this result, we suggest that decreased Notch-1 expression in myeloid cells reduces the PU.1 expression level, which impedes myeloid differentiation and induces AML.

Notch-1 regulates myeloid differentiation through increasing PU.1 mRNA expression levels; a high concentration of PU.1 is essential for myeloid differentiation. However, we speculated about a possible mechanism involving Notch-1/ PU.1 interaction other than transcriptional regulation. Coimmunoprecipitation of Notch-1 ICD and PU.1 in normal hematopoietic cells revealed that direct binding could occur between Notch-1 and PU.1. Decreased co-immunoprecipitation of Notch-1 ICD/PU.1 was found in AML cells (Fig. 3). We believe that Notch-1 regulates PU.1, not only at the transcriptional level, but also by direct interaction. After ligand binding of the Notch receptor, the ICD cleaves and binds to PU.1, which regulates its function. Partial disruption of the Notch-1 ICD/PU.1 complex might be involved in the leukemogenesis of AML.

The transcriptional factor PU.1 normally suppresses the development of myeloid leukemia; gene deletion and mutation of PU.1 impairs its DNA-binding ability, which induces leukemia in mice. Mutation of Notch-1 ICD also blocks the signal transduction. We therefore speculated that the mutation of Notch-1 ICD or PU.1 impedes the myeloid differentiation signals that cause leukemia. PCR-SSCP analysis was performed to detect possible mutations in the major regions of Notch-1 ICD (ANK, PEST) and in PU.1. No mutation was found in either the AML cell lines or in the patients. Thus, abnormal Notch signaling in AML might have been due to the down-regulation of Notch-1 expression, which reduces myeloid differentiation signaling, since there were no mutations that prohibited Notch signal transduction.

In conclusion, our novel study delineated the role of Notch-1 and myeloid differentiation factors PU.1 and MCSFR in AML patients. Down-regulation of Notch-1 expression reduced PU.1-mediated myeloid differentiation signaling in the AML patients and cell lines. Dual regulation of PU.1 by Notch-1 at the transcriptional level and by protein interaction was demonstrated in normal hematopoietic stem cells. Partial disruption of the Notch-1 ICD/PU.1 interaction might impair PU.1 function, which induces leukemia. No mutation of Notch-1 ICD and PU.1 was demonstrated in AML. These results suggest that down-regulation of Notch-mediated myeloid differentiation signaling might play an essential role in the development of AML.

\section{Acknowledgements}

This work was supported in part by Taipei Veterans General Hospital VGH (P.M.C), National Science Council of Taiwan (NSC 90-2314-B075-112) (P.M.C), and Taiwan Cancer Clinic Foundation. We thank Dr Masaki Hiramoto and Dr Aizawa Shin from Nihon University School of Medicine, Dr Yoshinobu Matsu from Fujisaki Cell Center and Hayashibara Biochemical Labs for providing the cell lines for our study.

\section{References}

1. Artavanis-Tsakonas S, Rand MD and Lake RJ: Notch signaling: cell fate control and signal integration in development. Science 284: 770-776, 1999.

2. Greenwald I: LIN-12/Notch signaling: lessons from worms and flies. Genes Dev 12: 1751-1762, 1998 .

3. Kimble J and Simpson P: The LIN-12/Notch signaling pathway and its regulation. Annu Rev Cell Dev Biol 13: 333-361, 1997.

4. Artavanis-Tsakonas S, Matsuno K and Fortini ME: Notch signaling. Science 268: 225-232, 1995.

5. Egan SE, St-Pierre B and Leow CC: Notch receptors, partners and regulators: from conserved domains to powerful functions. Curr Top Microbiol Immunol 228: 273-324, 1998.

6. de la Pompa JL, Wakeham A, Correia KM, et al: Conservation of the Notch signalling pathway in mammalian neurogenesis. Development 124:1139-1148, 1997.

7. Zhong W, Jiang MM, Weinmaster G, Jan LY and Jan YN: Differential expression of mammalian Numb, Numblike and Notch1 suggests distinct roles during mouse cortical neurogenesis. Development 124: 1887-1897, 1997.

8. Cossu G, Tajbakhsh S and Buckingham M: How is myogenesis initiated in the embryo? Trends Genet 12: 218-223, 1996.

9. Mumm JS and Kopan R: Notch signaling: from the outside in. Dev Biol 228: 151-165, 2000.

10. Milner LA and Bigas A: Notch as a mediator of cell fate determination in hematopoiesis: evidence and speculation. Blood 93: 2431-2448, 1999.

11. Kostyszyn B, Cowburn RF, Seiger A, Kjaeldgaard A and Sundstrom E: Expression of presenilin-1 and Notch-1 receptor in human embryonic CNS. Neuroscience 103: 885-898, 2001.

12. Allman D, Punt JA, Izon DJ, Aster JC and Pear WS: An invitation to $\mathrm{T}$ and more: notch signaling in lymphopoiesis. Cell 109: S1-S11, 2002.

13. Izon DJ, Punt JA and Pear WS: Deciphering the role of Notch signaling in lymphopoiesis. Curr Opin Immunol 14: 192-199, 2002 . 
14. Jaleco AC, Neves H, Hooijberg E, et al: Differential effects of Notch ligands Delta- 1 and Jagged-1 in human lymphoid differentiation. J Exp Med 194: 991-1002, 2001

15. Ellisen LW, Bird J, West DC, et al: TAN-1, the human homolog of the Drosophila notch gene, is broken by chromosomal translocations in T lymphoblastic neoplasms. Cell 66: 649-661, 1991.

16. Bresnick EH, Chu J, Christensen HM, Lin B and Norton J: Linking Notch signaling, chromatin remodeling, and T-cell leukemogenesis. J Cell Biochem 35: S46-S53, 2000.

17. Joutel A and Tournier-Lasserve E: Notch signaling pathway and human diseases. Semin Cell Dev Biol 9: 619-625, 1998.

18. Varnum-Finney B, Purton LE, Yu M, et al: The Notch ligand, Jagged-1, influences the development of primitive hematopoietic precursor cells. Blood 91: 4084-4091, 1998.

19. Jones P, May G, Healy L, et al: Stromal expression of Jagged 1 promotes colony formation by fetal hematopoietic progenitor cells. Blood 92: 1505-1511, 1998.

20. Tohda S and Nara N: Expression of Notch1 and Jagged1 proteins in acute myeloid leukemia cells. Leuk Lymphoma 42: 467-472, 2001.

21. Tohda S, Sakano S, Ohsawa M, Murakami N and Nara N: A novel cell line derived from de novo acute myeloblastic leukaemia with trilineage myelodysplasia which proliferates in response to a Notch ligand, Delta-1 protein. Br J Haematol 117: 373-378, 2002.

22. Pallisgaard N, Hokland P, Riishoj DC, Pedersen B and Jorgensen P: Multiplex reverse transcription-polymerase chain reaction for simultaneous screening of 29 translocations and chromosomal aberrations in acute leukemia. Blood 92: 574-588, 1998.

23. Mandelli F, Diverio D, Avvisati G, et al: Molecular remission in PML/RAR alpha-positive acute promyelocytic leukemia by combined all-trans retinoic acid and idarubicin (AIDA) therapy. Gruppo Italiano-Malattie Ematologiche Maligne dell'Adulto and Associazione Italiana di Ematologia ed Oncologia Pediatrica Cooperative Groups. Blood 90: 1014-1021, 1997.
24. Southern EM: Detection of specific sequences among DNA fragments separated by gel electrophoresis. J Mol Biol 98: 503-517, 1975.

25. Wu YY, Delgado RM, Sunderland T and Csako G: Semiautomated PCR-single-strand conformation polymorphism method for detection of a novel sequence polymorphism (Ile1000Val) in human alpha(2)-macroglobulin. Clin Chem 46: 715-718, 2000.

26. Zar JH: Chi-square correction for continuity. In: Biostatistical Analysis. Kurtz B (ed). 2nd edition, Prentice-Hall, Englewood Cliffs, NJ, pp48-53, 1984.

27. Zar JH: The Fisher exact test. In: Biostatistical Analysis. Kurtz B (ed). 2nd edition, Prentice-Hall, Englewood Cliffs, NJ, pp390-395, 1984

28. Kaplan EL and Meier P: Non-parametric estimation from incomplete observations. J Am Stat Assoc 53: 457-481, 1958.

29. Peto P and Pike MD: Conservatism of the approximation in the log-rank test for survival data or tumor incidence data. Biometrics 29: 579-584, 1973.

30. Schroeder T, Kohlhof H, Rieber N and Just U: Notch signaling induces multilineage myeloid differentiation and up-regulates PU.1 expression. J Immunol 170: 5538-5548, 2003.

31. Walker L, Carlson A, Tan-Pertel HT, Weinmaster G and Gasson J: The notch receptor and its ligands are selectively expressed during hematopoietic development in the mouse. Stem Cells 19: 543-552, 2001.

32. Morimura T, Goitsuka R, Zhang Y, Saito I, Reth M and Kitamura D: Cell cycle arrest and apoptosis induced by Notch1 in B cells. J Biol Chem 275: 36523-36531, 2000.

33. Schroeder T and Just U: mNotch1 signaling reduces proliferation of myeloid progenitor cells by altering cell-cycle kinetics. Exp Hematol 28: 1206-1213, 2000.

34. Ohishi K, Varnum-Finney B, Flowers D, Anasetti C, Myerson D and Bernstein ID: Monocytes express high amounts of Notch and undergo cytokine specific apoptosis following interaction with the Notch ligand, Delta-1. Blood 95: 2847-2854, 2000. 